\title{
CURVES WITH ONLY TRIPLE RAMIFICATION
}

\author{
STEFAN SCHRÖER
}

10 June 2002

\begin{abstract}
I show that the set of smooth curves of genus $g \geq 0$ admitting a branched covering $X \rightarrow \mathbb{P}^{1}$ with only triple ramification points is of dimension at least $\max (2 g-3, g)$. In characteristic two, such curves have tame rational functions and an analog of Belyi's Theorem applies to them.
\end{abstract}

\section{INTRODUCTION}

Let $C$ be a smooth proper curve over an algebraically closed ground field $k$ of arbitrary characteristic $p \geq 0$. Fulton [3] showed that there are always generically étale finite maps $C \rightarrow \mathbb{P}^{1}$ whose ramification points have index $e=2$. In this paper, I pose the following question: Does there exist a generically étale finite map $C \rightarrow \mathbb{P}^{1}$ whose ramification points all have index $e=3$ ?

Fried, Klassen, and Kopeliovich [2] took a first step into this direction. They proved that all but finitely many complex elliptic curves admit such a map. In fact, their proof reveals that for any given genus $g \geq 1$, the set of Riemann surfaces of genus $g$ admitting such maps is at least 1-dimensional. The arguments, however, are purely topological and involve homeomorphism spaces, Dehn twists, and Teichmüller theory.

The main result of this paper is an improved lower bound on the dimension via purely algebraic methods. We shall prove that the set of points in $M_{g}$ whose corresponding curve admits rational functions with only triple ramification has dimension $\geq \max (2 g-3, g)$. Our arguments work in all characteristics $p \neq 3$ and rely on deformation theory and the moduli space of stable curves. The basic idea is to deform a covering $X_{0} \rightarrow \mathbb{P}^{1}$ where $X_{0}$ is a curve with cuspidal singularities, so that each cuspidal ramification point breaks up into two regular ramification points. The semistable reduction involved in this neatly explains why we miss $g$ dimensions from the $(3 g-3)$-dimensional moduli space $M_{g}$. One might speculate whether or not we found the best lower bound.

My motivation to study this problem is Belyi's Theorem 11. It states that a compact Riemann surface is defined over a number field if and only if it admits a finite map to the Riemann sphere with at most three branch points. Saïdi [11] generalized this to odd characteristics as follows: An algebraic curve $C$ in characteristic $p \geq 3$ is defined over a finite field if and only if it admits a tamely ramified morphism $C \rightarrow \mathbb{P}^{1}$ with at most three branch points. In characteristic $p=2$, the if part holds true, but the only-if part remains mysterious. However, a curve $C$ over $\bar{F}_{2}$ admits a tame function with at most three branch points if it admits a tame

1991 Mathematics Subject Classification. 14H10, 14H25, $14 \mathrm{H} 51$.

Key words and phrases. triple ramification, tame coverings, Belyi's Theorem. 
function at all. In some sense, the result of this paper tells us that the Belyi-Saïdi Theorem is valid in characteristic $p=2$ at least for a $(2 g-3)$-dimensional set.

The question whether a finite morphism $X \rightarrow \mathbb{P}^{1}$ whose ramification points have index $e=3$ exists is also interesting for nonclosed ground fields. There, however, I showed in 12 that the generic curve $C_{\eta}$ of genus $g \geq 3$ in characteristic $p=2$ does not admit such a map. This relies on Franchetta's Conjecture, which states that $\operatorname{Pic}\left(C_{\eta}\right)=\mathbb{Z} K_{C_{\eta}}$. Here the ground field is the function field $\kappa(\eta)$ of the moduli space $M_{g}$. Of course, it still might be true that the desired map exists over some field extension $\kappa(\eta) \subset L$.

Here is a plan for the paper. In Section 1 we study collisions of triple ramification points in terms of local equations and semistable reduction. To globalize this, we collect in Section 2 some general results about deformations of coverings whose fibers are complete intersections. We use this to construct effective formal deformation in Section 3, and explain the resulting increase of transcendence degree in moduli fields. Section 4 contains a construction of maps $\mathbb{P}^{1} \rightarrow \mathbb{P}^{1}$ with only triple coverings so that $\mathbb{P}^{1}$ marked with the ramification points has a large moduli field. We use this to prove our main result, which occurs in Section 5. The last section contains some applications regarding Belyi's Theorem in positive characteristics.

Acknowledgement. I thank Tamás Szamuely, János Kollár, Fabrizio Catanese, and Hubert Flenner for helpful discussions, and Le Van Ly for careful proof-reading.

\section{Collision of triple RAMification POINTS}

The key idea of this paper is to collide triple ramification points. To explain this, let me start with the complex-analytic viewpoint.

Let $D=\{z \in \mathbb{C}|| z \mid<1\}$ be the complex unit disc. For each nonzero $t \in D$, the fundamental group $\pi_{1}(D-\{0, t\})=\mathbb{Z} * \mathbb{Z}$ is a free group on two generators, and étale triple coverings $X^{*} \rightarrow D-\{0, t\}$ correspond to the choice of two permutations $\sigma_{0}, \sigma_{t} \in S_{3}$ specifying the local monodromies. Moreover, such étale coverings extend uniquely to branched coverings $X \rightarrow D$. What happens if $t \rightarrow 0$, that is, if the branch points collide? We shall analyze this now in the case that both permutations $\sigma_{0}$ and $\sigma_{t}$ equal the 3 -cycle $(1,2,3)$.

The equation $u^{3}-z(z-t)=0$ defines a closed subspace $X_{t} \subset \mathbb{C} \times D$ depending on a parameter $t$. For $t \neq 0$, the canonical projection $f_{t}: X_{t} \rightarrow D$ is a branched covering with branch points $0, t \in D$. The covering is connected, because the polynomial $u^{3}-z(z-t)$ is irreducible. Clearly, the local monodromies $\sigma_{0}, \sigma_{t}$ are 3 -cycles. The product $\sigma_{0} \sigma_{t}$, which is the monodromy at infinity, is a 3 -cycle as well. To see this, calculate the winding number of $z(z-t)$ around $0 \in \mathbb{C}$, for $z=(1+t) e^{2 \pi i r}$ with $0 \leq r \leq 1$. The winding number in question is

$$
\frac{1}{2 \pi i} \int_{z^{2}-z t} \frac{d u}{u}=\frac{1}{2 \pi i} \int_{(1+t) e^{2 \pi i r}} \frac{2 z-t}{z(z-t)} d z=2
$$

by residue calculus. It follows that $\sigma_{0} \sigma_{t}$ has order three, so after a suitable enumeration of sheets our monodromy permutations are $\sigma_{0}=\sigma_{t}=(1,2,3)$.

In the limit $t \rightarrow 0$, the defining equation becomes $u^{3}-x^{2}=0$, so $X_{0} \subset \mathbb{C} \times D$ is a curve with a cuspidal singularity. The completion of the local ring is

$$
\mathcal{O}_{X_{0}, 0}^{\wedge}=\mathbb{C}[[u, z]] /\left(u^{3}-z^{2}\right) \simeq \mathbb{C}\left[\left[s^{2}, s^{3}\right]\right],
$$


the latter isomorphism given by $u=s^{2}$ and $z=s^{3}$. The normalization $\tilde{X}_{0} \rightarrow X_{0}$ comes from the inclusion $\mathbb{C}\left[\left[s^{2}, s^{3}\right]\right] \subset \mathbb{C}[[s]]$. Hence the induced covering $\tilde{X}_{0} \rightarrow D$ is a triple covering with one ramification point, which has ramification index $e=3$.

We now switch to an algebraic viewpoint. Fix a ground field $k$ of characteristic $p \neq 3$, and set $A=k[[t]]$ and $A_{n}=A /\left(t^{n+1}\right)$. We shall use $t$ as a parameter for deformations. Consider the $A$-algebras $B=k[[z, t]]$ and $B_{n}=B \otimes_{A} A_{n}$. Then $B_{0}=k[[z]]$, and $B_{n}=B_{0} \otimes_{k} A_{n}$, and $B=\varliminf_{\longleftrightarrow} B_{n}$. Set

$$
C=k[[z, t, u]] /\left(u^{3}-z(z-t)\right) \quad \text { and } \quad C_{n}=C \otimes_{A} A_{n} .
$$

Then $C$ is a free $B$-module with basis $1, u, u^{2}$.

Set $S=\operatorname{Spec}(A)$, and $Y=\operatorname{Spec}(B)$, and $X=\operatorname{Spec}(C)$. Similarly, we define $S_{n}=\operatorname{Spec}\left(A_{n}\right)$ et cetera. Then $X_{0}=\operatorname{Spec}\left(C_{0}\right)$ is a complete local 1-dimensional scheme with cuspidal singularity. The canonical morphism $f_{0}: X_{0} \rightarrow Y_{0}$ is finite and flat of degree three. We now view $f_{n}: X_{n} \rightarrow Y_{n}$ as infinitesimal deformation of $f_{0}$ parameterized by $S_{n}$. The collection $\left(f_{n}\right)_{n \geq 0}$ defines a formal deformation over the formal spectrum $\mathfrak{S}=\operatorname{Spf}(A)$. By construction, this formal deformation is effective, that is, it comes from a morphism $f: X \rightarrow Y$ of schemes over $S$.

Let $\eta \in S$ be the generic point, whose residue field $\kappa(\eta)$ is the field of formal Laurent series $k((t))=k[[t]][1 / t]$. The generic fiber $Y_{\eta}=\operatorname{Spec} B[1 / t]$ is a regular 1-dimensional scheme, whose closed points correspond to the primes of height one in $k[[z, t]]$ different from $(t)$. Let $b, b^{\prime} \in Y_{\eta}$ be the closed points corresponding to the primes $(z)$ and $(z-t)$, respectively. Then $f_{\eta}: X_{\eta} \rightarrow Y_{\eta}$ is a triple covering with $b$ and $b^{\prime}$ as branch points. The ramification is total over each branch point.

The closed fiber $X_{0}$ of the family $X \rightarrow S$ has a cuspidal singularity. However, we may achieve semistable reduction after suitable base change. More precisely:

Proposition 1.1. The family of formal curves $X \rightarrow S$ admits a semistable reduction $X^{\prime}$ after base-changing along $t \mapsto t^{3}$. The proper part of $X_{0}^{\prime}$ is an elliptic curve with $j=0$. It intersects the nonproper part of $X_{0}^{\prime}$ in a single point.

Proof. Let $Z \rightarrow X$ be the blowing-up of the closed point. To calculate this, consider the blowing-up of $k[u, z, t]$ at the origin. This is given by the three charts $k[u / t, z / t, t]$, and $k[u / z, z, t / z]$, and $k[u, z / u, t / u]$. The strict transform of $X$ has equations $t(u / z)^{3}-(z / t)^{2}+z / t=0$, and $z(u / z)^{3}-1+t / z=0$, and $u-(z / u)^{2}+(z / u)(t / u)=0$, respectively. It follows that $Z$ is regular. Setting $u=z=t=0$, we see that the exceptional divisor for $Z \rightarrow X$ is a reducible quadric $Q \subset \mathbb{P}_{k}^{2}$, which intersects the nonproper part of $Z_{0}$ in the double point. Blowing up this triple intersection, we obtain a configuration

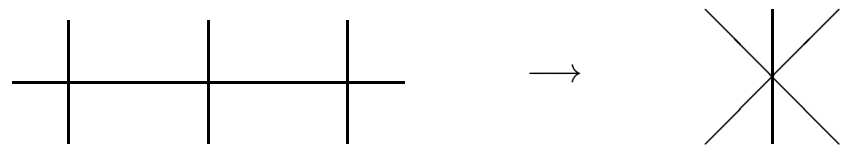

where the horizontal $\mathbb{P}_{k}^{1}$ has multiplicity three, and the three vertical components have multiplicity one.

To achieve semistable reduction, we follow the algorithm given in [8], page 122130. In particular, the normalized base change $X^{\prime}$ along $t \mapsto t^{3}$ is nothing but the branched covering of degree three branched along the three vertical components with multiplicity one. Like on page 129 , we conclude that the proper part of $X_{0}^{\prime}$ is an elliptic curve with $j$-invariant $j=0$, and it intersects the nonproper part of $X_{0}^{\prime}$ 
in a single point. Note that the algorithm in [8] is stated over the complex numbers. However, it remains valid in characteristic $p \neq 3$, because it involves only maps of degree three.

Remark 1.2. Unfortunately, the elliptic curve $E$ occurring in the semistable reduction is not generic. This explains why in Theorem 5.1, we only get moduli fields of transcendence degree at least $\max (2 g-3, g)$, and not the maximal possible transcendence degree $3 g-3$.

\section{Deformations For COVERINGS OF COMPLETE INTERSECTION}

Fix a ground field $k$ of arbitrary characteristic $p \geq 0$. Let $X$ and $Y$ be two curves without embedded components, and $f: X \rightarrow Y$ a flat finite morphism that is generically étale. Then $\Omega_{X / Y}^{1}$ is a coherent skyscraper sheaf supported by the ramification points $x \in X$. We shall study infinitesimal deformations of $f: X \rightarrow Y$. Let $R$ be a local Artin $k$-algebra with residue field $k$. A deformation of $f$ over $A$ consists of a curve $\mathfrak{X}_{A}$ flat and of finite type over $A$, a morphism $g_{A}: \mathfrak{X}_{A} \rightarrow Y \otimes A$, and an isomorphism $g_{A} \otimes_{A} k \simeq g$.

Suppose $I \subset A$ is an ideal with $I^{2}=0$, and $g_{A / I}: \mathfrak{X}_{A / I} \rightarrow Y \otimes A / I$ is a deformation over $A / I$. According to [9], Proposition 2.1.2.3, the obstruction for extending it to a deformation $g_{A}: \mathfrak{X}_{A} \rightarrow Y \otimes A$ over $A$ lies in the vector space of hyperextensions

$$
\operatorname{Ext}^{2}\left(L_{X / Y}^{\bullet}, \mathcal{O}_{X} \otimes_{k} I\right)=\mathbb{E x t}^{2}\left(L_{X / Y}^{\bullet}, \mathcal{O}_{X}\right) \otimes_{k} I
$$

Here $L_{X / Y}^{\bullet}$ is the cotangent complex for $f$. These obstructions vanish under suitable assumptions. Recall that $f: X \rightarrow Y$ is a morphism of complete intersection if for all $x \in X$, the Artin local $\operatorname{ring} \mathcal{O}_{X, x} / \mathfrak{m}_{f(x)} \mathcal{O}_{X, x}$ is the quotient of some power series algebra $\kappa(x)\left[\left[t_{1}, \ldots, t_{n}\right]\right]$ by a regular sequence ([7], Definition 19.3.6).

Proposition 2.1. If the morphism of curves $f: X \rightarrow Y$ is of complete intersection, then the group $\mathbb{E x t}^{2}\left(L_{X / Y}^{\bullet}, \mathcal{O}_{X}\right)$ vanishes.

Proof. Consider the spectral sequence $\operatorname{Ext}^{r}\left(\mathcal{H}_{s}\left(L_{X / Y}^{\bullet}\right), \mathcal{O}_{X}\right) \Rightarrow \operatorname{Ext}^{r+s}\left(L_{X / Y}^{\bullet}, \mathcal{O}_{X}\right)$. The sheaves $\mathcal{H}_{s}\left(L_{X / Y}^{\bullet}\right)$ are skyscraper sheaves supported by the ramification points $x \in X$. We have $\operatorname{Ext}^{0}\left(\mathcal{H}_{s}\left(L_{X / Y}^{\bullet}\right), \mathcal{O}_{X}\right)=0$ because $X$ has no embedded components. Moreover, we have $\mathcal{H}_{1}\left(L_{X / Y}^{\bullet}\right)=0$. To see this, fix a ramification point $x \in X$, and write $A=\mathcal{O}_{Y, f(x)}^{\wedge}$ and $B=\mathcal{O}_{X, x}^{\wedge}$. Then we have $B=C / I$ for some power series algebra $C=A\left[\left[t_{1}, \ldots, t_{n}\right]\right]$ with $n$ sufficiently large. According to [7], Corollary 19.3.5, the ideal $I \subset C$ is generated by a regular sequence, because $f$ is a morphism of complete intersection. Therefore the $B$ module $I / I^{2}$ is free. Moreover, the cotangent complex $L_{B / A}^{\bullet}$ is quasiisomorphic to $I / I^{2} \rightarrow \Omega_{C / A}^{1} \otimes B$. The map $I / I^{2} \rightarrow \Omega_{C / A}^{1} \otimes B$ is generically bijective, because $f$ is generically étale. Hence its kernel vanishes, since $\mathcal{O}_{X}$ has no embedded components. In turn, $\mathcal{H}_{1}\left(L_{X / Y}^{\bullet}\right)_{x}=H_{1}\left(L_{B / A}^{\bullet}\right)$ vanishes. Summing up, the edge map

$$
\operatorname{Ext}^{2}\left(\Omega_{X / Y}^{1}, \mathcal{O}_{X}\right) \longrightarrow \mathbb{E x t}^{2}\left(L_{X / Y}^{\bullet}, \mathcal{O}_{X}\right)
$$

from the spectral sequence is surjective. Next, consider the spectral sequence

$$
H^{r}\left(Y, \mathcal{E} x t^{s}\left(\Omega_{X / Y}^{1}, \mathcal{O}_{X}\right)\right) \Longrightarrow \operatorname{Ext}^{r+s}\left(\Omega_{X / Y}^{1}, \mathcal{O}_{X}\right)
$$


The sheaf $\mathcal{E} x t^{0}\left(\Omega_{X / Y}^{1}, \mathcal{O}_{X}\right)$ vanishes, because $\Omega_{X / Y}^{1}$ is torsion and $\mathcal{O}_{X}$ is torsion free. The group $H^{1}\left(Y, \mathcal{E} x t^{1}\left(\Omega_{X / Y}^{1}, \mathcal{O}_{X}\right)\right)$ vanishes because $\mathcal{E} x t^{1}\left(\Omega_{X / Y}^{1}, \mathcal{O}_{X}\right)$ has 0 dimensional support. The sheaf $\mathcal{E} x t^{2}\left(\Omega_{X / Y}^{1}, \mathcal{O}_{Y}\right)$ vanishes, because the stalks of $\Omega_{X / Y}^{1}$ have projective dimension $\leq 1$ by the local arguments above. We conclude that $\operatorname{Ext}^{2}\left(\Omega_{X / Y}^{1}, \mathcal{O}_{X}\right)$ and hence $\operatorname{Ext}^{2}\left(L_{X / Y}^{\bullet}, \mathcal{O}_{X}\right)$ vanish.

Suppose again that $I \subset A$ has square zero, and that $g_{A / I}: \mathfrak{X}_{A / I} \rightarrow Y \otimes A / I$ is a deformation over $A / I$. According to [9], Proposition 2.1.2.3, the set of isomorphism classes of deformation $g_{R}: \mathfrak{X}_{A} \rightarrow Y \otimes A$ endowed with an isomorphism $g_{A} \otimes A / I \simeq$ $g_{A / I}$ is an affine space for the vector space of hyperextensions

$$
\operatorname{Ext}^{1}\left(L_{X / Y}^{\bullet}, \mathcal{O}_{X} \otimes_{k} I\right)=\operatorname{Ext}^{1}\left(L_{X / Y}^{\bullet}, \mathcal{O}_{X}\right) \otimes_{k} I .
$$

This group splits up into complete local parts:

Proposition 2.2. Let $x_{i} \in X$ be the ramification points and $y_{i}=f\left(x_{i}\right)$ the corresponding branch points. Then $\operatorname{Ext}^{1}\left(L_{X / Y}^{\bullet}, \mathcal{O}_{X}\right) \simeq \bigoplus_{i} \operatorname{Ext}^{1}\left(\Omega_{\mathcal{O}_{\hat{X}, x_{i}}}^{1} / \mathcal{O}_{\hat{Y}, y_{i}}, \mathcal{O}_{X, x_{i}}^{\wedge}\right)$.

Proof. The spectral sequence $\operatorname{Ext}^{r}\left(\mathcal{H}_{s}\left(L_{X / Y}^{\bullet}\right), \mathcal{O}_{X}\right) \Rightarrow \operatorname{Ext}^{r+s}\left(L_{X / Y}^{\bullet}, \mathcal{O}_{X}\right)$ gives an exact sequence

$$
0 \longrightarrow \operatorname{Ext}^{1}\left(\Omega_{X / Y}^{1}, \mathcal{O}_{X}\right) \longrightarrow \operatorname{Ext}^{1}\left(L_{X / Y}^{\bullet}, \mathcal{O}_{X}\right) \longrightarrow \operatorname{Hom}\left(\mathcal{H}_{1}\left(L_{X / Y}^{\bullet}\right), \mathcal{O}_{X}\right) .
$$

The term on the right vanishes because $\mathcal{H}_{1}\left(L_{X / Y}^{\bullet}\right)$ is torsion and $\mathcal{O}_{X}$ is torsion free. The coherent $\mathcal{O}_{X}$-module $\Omega_{X / Y}^{1}$ is a skyscraper sheaf supported by the $x_{i} \in X$, so $\Omega_{X / Y}^{1}=\bigoplus_{i} \Omega_{\mathcal{O}_{\hat{X}, x_{i}}}^{1} / \mathcal{O}_{\hat{Y}, y_{i}}$ and the result follows.

\section{Construction of effective formal Deformations}

We now apply the results of the preceding section in the following situation. Fix a ground field $k$ of characteristic $p \neq 3$, and let $\tilde{X}_{0}$ be a smooth proper geometrically connected curve of genus $g \geq 0$ over $k$. Suppose we have a finite generically étale morphism $\tilde{f}: \tilde{X}_{0} \rightarrow \mathbb{P}_{k}^{1}$ whose ramification points are all rational and have index $e=3$. In this situation we also say that $\tilde{f}$ has only triple ramification.

Let $\tilde{x} \in \tilde{X}_{0}$ be such a ramification point. Applying an automorphism of $\mathbb{P}_{k}^{1}$, we may assume that $\tilde{x}$ maps to $0 \in \mathbb{P}_{k}^{1}$. Choose uniformizers $s \in \mathcal{O}_{\tilde{X}_{0}, \tilde{x}}$ and $z \in \mathcal{O}_{\mathbb{P}_{k}^{1}, 0}$ with $z \mapsto s^{3}$. Let $X_{0}$ be the singular curve of arithmetic genus $g+1=h^{1}\left(\mathcal{O}_{X_{0}}\right)$ defined by the cocartesian square

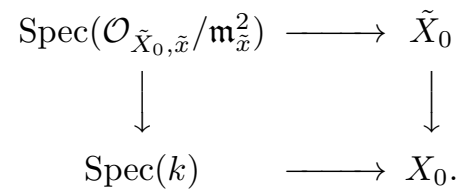

Then $\tilde{X}_{0} \rightarrow X_{0}$ is the normalization, and the image $x \in X_{0}$ of $\tilde{x} \in \tilde{X}_{0}$ is a cuspidal singularity with $\mathcal{O}_{X_{0}, x}^{\wedge}=k\left[\left[s^{2}, s^{3}\right]\right]$. Since $\tilde{x}$ is a ramification point, the morphism $\tilde{f}_{0}: \tilde{X}_{0} \rightarrow \mathbb{P}_{k}^{1}$ induces a finite generically étale morphism $f_{0}: X_{0} \rightarrow \mathbb{P}_{k}^{1}$ with $f(x)=0$. The map of local rings $\mathcal{O}_{\mathbb{P}_{k}^{1}, 0} \rightarrow \mathcal{O}_{X_{0}, x}^{\wedge}$ is given by $z \mapsto s^{3}$. Obviously, the flat morphism $f_{0}: X_{0} \rightarrow \mathbb{P}_{k}^{1}$ is of complete intersection.

Proposition 3.1. Set $A=k[[t]]$. Then there is a flat family $X \rightarrow \operatorname{Spec}(A)$ and $a$ finite A-morphism $f: X \rightarrow \mathbb{P}_{A}^{1}$ such that the following holds: 
(i) The closed fiber $f \otimes k$ is isomorphic to $f_{0}: X_{0} \rightarrow \mathbb{P}_{k}^{1}$.

(ii) The formal completion $\mathcal{O}_{X}^{\wedge}$ is isomorphic to $k[[u, z, t]] /\left(u^{3}-z(z-t)\right)$ as algebra over $\mathcal{O}_{\mathbb{P}_{A}^{1}, 0}^{\wedge}=k[[z, t]]$, like in Section $\square$.

(iii) For every closed point $y \in X_{0}$ with $y \neq x$, the formal completion $\mathcal{O}_{X, y}^{\wedge}$ is isomorphic to $\mathcal{O}_{X_{0}, y}^{\wedge}[[t]]$ as algebra over $\mathcal{O}_{\mathbb{P}_{A}^{1}, f(y)}^{\wedge}=\mathcal{O}_{\mathbb{P}_{k}^{1}, f(y)}^{\wedge}[[t]]$.

(iv) The generic fiber $X_{\eta}$ is a smooth geometrically connected curve of genus $g+1$.

Proof. First, we shall construct a formal flat morphism $\mathfrak{X} \rightarrow \operatorname{Spf}(A)$ and a finite formal morphism $f: \mathfrak{X} \rightarrow \mathbb{P}_{k}^{1} \times \operatorname{Spf}(A)$ with corresponding properties (i)-(iii). Set $A_{n}=A /\left(t^{n+1}\right)$. Suppose we already have constructed a flat $A_{n-1}$-scheme $X_{n-1}$ and a morphism $f_{n-1}: X_{n-1} \rightarrow \mathbb{P}_{A_{n-1}}^{1}$ with properties as in (i)-(iii). According to Proposition 2.1, there is a flat $A_{n}$-scheme $X_{n}^{\prime}$ and a morphism $f_{n}^{\prime}: X_{n} \rightarrow \mathbb{P}_{A_{n}}^{1}$ whose restriction to $A_{n-1}$ admits an isomorphism $\varphi_{n}^{\prime}: f_{n}^{\prime} \otimes A_{n-1} \simeq f_{n-1}$. The set of isomorphism classes of such extensions is a torsor under

$$
\bigoplus_{i} \operatorname{Ext}^{1}\left(\Omega_{\mathcal{O}_{\hat{X}_{0}, x_{i}}}^{1} / \mathcal{O}_{\mathbb{P}_{k}^{1}, y_{i}}^{\wedge}, \mathcal{O}_{X_{0}, x_{i}}^{\wedge}\right)
$$

by Proposition 2.2, where $x_{i} \in X$ are the ramification points and $y_{i} \in \mathbb{P}_{k}^{1}$ are the corresponding branch points. Hence we may choose, for each ramification point $x_{i} \in X$, an element $\alpha_{i} \in \operatorname{Ext}^{1}\left(\Omega_{\mathcal{O}_{\hat{X}_{0}, x_{i}} / \mathcal{O}_{\mathbb{P}_{k}^{1}, y_{i}}^{\wedge}}^{1}, \mathcal{O}_{X_{0}, x_{i}}^{\wedge}\right)$ so that $\left(\alpha_{i}\right)$ applied to the isomorphism class of $\left(X_{n}^{\prime}, f_{n}^{\prime}, \varphi_{n}^{\prime}\right)$ gives another flat $A_{n}$-scheme $X_{n}$ together with a morphism $f_{n}: X_{n} \rightarrow \mathbb{P}_{A_{n}}^{1}$ satisfying our conditions (i)-(iii). By induction we construct for all integers $n \geq 0$ morphisms $f_{n}: X_{n} \rightarrow \mathbb{P}_{A_{n}}^{1}$ together with identifications $\varphi_{n}: f_{n} \otimes A_{n-1} \simeq f_{n-1}$. Such a system is nothing but the desired morphism of formal schemes.

Being 1-dimensional, the proper scheme $X_{0}$ admits an ample invertible sheaf. The obstruction for extending an invertible sheaf from $X_{n-1}$ to $X_{n}$ lies in the group $H^{2}\left(X_{0}, \mathcal{O}_{X_{0}}\right)=0$. It follows that there is a formal invertible sheaf on $\mathfrak{X}$ that is ample on $X_{0}$. By Grothendieck's algebraization theorem ([4], Theorem 5.4.5), the formal scheme $\mathfrak{X}$ is the formal completion of a projective $A$-scheme $X$. Moreover (陆, Theorem 5.4.1), the morphism $\mathfrak{X} \rightarrow \mathbb{P}^{1} \times \operatorname{Spf}(A)$ of formal schemes comes from a morphism of schemes $f: X \rightarrow \mathbb{P}_{A}^{1}$. Properties (i)-(iii) hold because they depend only on the underlying formal schemes.

Concerning the last property (iv), observe that $X_{0}$ is geometrically integral, because $\tilde{X}_{0} \rightarrow X_{0}$ is a birational universal homeomorphism. Then $X_{\eta}$ is geometrically integral as well by [6], Theorem 12.2.1. Hence $h^{0}\left(\mathcal{O}_{X_{0}}\right)=h^{0}\left(\mathcal{O}_{X_{\eta}}\right)$ and in turn $g+1=h^{1}\left(\mathcal{O}_{X_{0}}\right)=h^{1}\left(\mathcal{O}_{X_{\eta}}\right)$ by flatness.

Starting with a smooth proper geometrically connected curve $\tilde{X}_{0}$ over a field $k$ of genus $g \geq 1$ endowed with a finite generically étale morphism $\tilde{X}_{0} \rightarrow \mathbb{P}_{k}^{1}$ with only triple ramification, Proposition 3.1 produces a smooth proper geometrically connected curve $X_{\eta}$ over $k((t))$ of genus $g+1$ endowed with a finite generically étale morphism $X_{\eta} \rightarrow \mathbb{P}_{\eta}^{1}$ with only triple ramification. The curve $X_{\eta}$ defines a morphism Spec $k((T)) \rightarrow M_{g+1}$ into the moduli space of smooth stable curves. Let $x \in M_{g+1}$ be the image point. Its residue field $\kappa(x)$ is called the moduli field for the smooth stable curve $X_{\eta}$.

Proposition 3.2. The moduli field for the smooth stable curve $X$ has transcendence degree $\geq 1$ over $k$. 
Proof. Consider the moduli space $\bar{M}_{g+1}$ of stable curves of genus $g+1$. According to Proposition 1.1, the semistable reduction $X^{\prime} \rightarrow \operatorname{Spec}\left(A^{\prime}\right)$ for $X \rightarrow \operatorname{Spec}(A)$ defines a morphism $\operatorname{Spec}\left(A^{\prime}\right) \rightarrow \bar{M}_{g+1}$ not factoring over the open subset $M_{g+1}$. Recall that $A=k[[t]]$. We conclude that the image $x \in M_{g+1}$ of $\eta \in \operatorname{Spec}(A)$ is not a closed point, and the statement follows.

\section{Moduli fields FOR POINTED RATiOnal CURVES}

Fix a ground field $k$ of characteristic $p \neq 3$, and let $n \geq 3$ be an integer. In this section we study stable $n$-pointed curves $\left(\mathbb{P}_{K}^{1}, x_{1}, \ldots, x_{n}\right)$ defined over field extensions $k \subset K$ and their moduli fields. Note that the corresponding moduli space $M_{0, n}$ is fine, because any automorphism of $\mathbb{P}_{K}^{1}$ fixing three rational points is the identity.

To calculate moduli fields, we first apply some $\gamma \in \mathrm{PGL}_{2}(K)$ to obtain $x_{0}=0$, $x_{1}=\infty$, and $x_{2}=1$. Then the moduli field for $\left(\mathbb{P}_{K}^{1}, x_{1}, \ldots, x_{n}\right)$ is precisely the field generated by the fields of definition for the remaining marked points $x_{4}, \ldots, x_{n} \in$ $\mathbb{P}_{K}^{1}$ viewed as closed subschemes (compare [5], Corollary 4.8.11). It follows from this description that the moduli field neither changes under permuting the marked points nor under applying an automorphism $\gamma \in \mathrm{PGL}_{2}(K)$.

Now suppose we have a stable curve $\left(\mathbb{P}_{K}^{1}, x_{1}, \ldots, x_{n}\right)$ and a finite group $G$ acting on $\mathbb{P}_{K}^{1}$ such that each orbit contains at most one marked point. Then the quotient is $\mathbb{P}_{K}^{1} / G \simeq \mathbb{P}_{K}^{1}$, and the images $y_{i} \in \mathbb{P}_{K}^{1}$ of the marked points $x_{i} \in \mathbb{P}_{K}^{1}$ define another stable curve $\left(\mathbb{P}_{K}^{1}, y_{1}, \ldots, y_{n}\right)$.

Proposition 4.1. Suppose $G=\mathbb{Z} / l \mathbb{Z}$ is cyclic of order prime to $p$. Assume that one fixed point for the action is rational and marked. Then the moduli fields for $\left(\mathbb{P}_{K}^{1}, x_{1}, \ldots, x_{n}\right)$ and $\left(\mathbb{P}_{K}^{1}, y_{1}, \ldots, y_{n}\right)$ have the same transcendence degree over $k$.

Proof. We may assume that the group action is faithful. The generator $g \in G$ acts via some $A \in \mathrm{GL}_{2}(K)$. This matrix has an eigenvector because the $G$-action has a fixed point, which is rational by assumption. If $A$ has no other eigenvector, the matrix is conjugate to $\left(\begin{array}{ll}\xi & \lambda \\ 0 & \xi\end{array}\right)$, where $\lambda \in K$ is a scalar with $l \lambda=0$, which is impossible because $l$ is prime to the characteristic.

Hence $A$ is diagonizable, so we may assume that $g \in G$ acts via $\left(\begin{array}{ll}1 & 0 \\ 0 & \xi\end{array}\right)$ for some primitive $l$-th root of unity $\xi \in k$. The upshot is that the $G$-action is defined over the moduli field of $\left(\mathbb{P}_{K}^{1}, x_{1}, \ldots, x_{n}\right)$, and so is the quotient $\left(\mathbb{P}_{K}^{1}, y_{1}, \ldots, y_{n}\right)$.

Conversely, let $L$ be the moduli field of $\left(\mathbb{P}_{K}^{1}, y_{1}, \ldots, y_{n}\right)$. As above, the quotient map $f: \mathbb{P}_{K}^{1} \rightarrow \mathbb{P}_{K}^{1} / G \simeq \mathbb{P}_{K}^{1}$ is defined over $L(\xi)$, and the marked points $x_{i} \in \mathbb{P}_{K}^{1}$ are defined over some finite field extension of $L(\xi)$ generated by the residue fields from the fibers $f^{-1}\left(y_{i}\right)$.

Now assume that there are algebraically independent elements $t_{4}, \ldots, t_{n} \in K$, and consider the $n$-pointed stable curve $\left(\mathbb{P}_{K}^{1}, y_{1}, \ldots, y_{n}\right)$ with $y_{1}=0, y_{2}=\infty$, $y_{3}=1$, and $y_{i}=t_{i}$ for $4 \leq i \leq n$. Its field of definition is the purely transcendental field extension $k\left(t_{4}, \ldots, t_{n}\right) \subset K$, which has transcendence degree $n-3$.

Proposition 4.2. There is a finite field extension $K \subset L$ and a generically étale finite map $f: \mathbb{P}_{L}^{1} \rightarrow \mathbb{P}_{L}^{1}$ with only triple ramification such that the $y_{i} \in \mathbb{P}_{L}^{1}$ occur as branch points. If we choose for each $y_{i}$ a ramification point $x_{i} \in f^{-1}\left(y_{i}\right)$, then the moduli field for the $\left(\mathbb{P}_{L}^{1}, x_{1}, \ldots, x_{n}\right)$ has transcendence degree $n-3$. 
Proof. Consider the polynomial map $g: \mathbb{P}_{K}^{1} \rightarrow \mathbb{P}_{K}^{1}$ given by $z \mapsto z^{3}$ on the affine part $\mathbb{A}_{K}^{1} \subset \mathbb{P}_{K}^{1}$. Then $g$ is a generically étale finite map of degree three with only triple ramification, whose ramification and branch points are $0, \infty \in \mathbb{P}_{K}^{1}$. For suitable $\lambda \in \mathrm{PGL}_{2}(K)$, the composition $\lambda g$ realizes any given pair of rational points $a, b \in \mathbb{P}_{K}^{1}$ as branch locus.

We now construct the desired map $f$ by induction. Suppose we already have a generically étale finite map $f_{i}: \mathbb{P}_{L_{i}}^{1} \rightarrow \mathbb{P}_{L_{i}}^{1}$ with only triple ramification such that $y_{j}$ occurs as branch points for $1 \leq j \leq i$ and $f_{i}$ is étale over $y_{j}$ for $i+1 \leq j \leq n$. Let $L_{i} \subset L^{\prime}$ be a finite field extension such that there is a rational point $x^{\prime} \in f_{i}^{-1}\left(y_{i+1}\right)$. Choose $\lambda_{i} \in \mathrm{PGL}_{2}(K)$ so that $x^{\prime}$ is a branch point for $\lambda_{i} g$, but that $\lambda_{i} g$ is étale over $f_{i}^{-1}\left(y_{j}\right)$ for $j \neq i+1$. Then $f_{i+1}=f_{i} \lambda_{i} g$ is the desired map. As for the ground field, choose a finite field extension $L_{i} \subset L_{i+1}$ so that the fibers of $f_{i+1}: \mathbb{P}_{L_{i+1}}^{1} \rightarrow \mathbb{P}_{L_{i+1}}^{1}$ over the branch points $y_{j}$ contain only rational points.

It remains to check the assertion on moduli fields. We shall prove by induction on $i \geq 0$ that for each choice of rational points $x_{j} \in f_{i}^{-1}\left(y_{j}\right)$ for $1 \leq j \leq n$ such that $x_{j}$ with $1 \leq j \leq i$ are ramification points for $f_{i}$, the moduli field of $\left(\mathbb{P}_{L_{n}}^{1}, x_{1}, \ldots, x_{n}\right)$ has transcendence degree $n-3$. Set $x_{j}^{\prime}=\lambda_{i}\left(g\left(x_{j}\right)\right)$. Inductively we know that the moduli field of $\left(\mathbb{P}_{L_{n}}^{1}, x_{1}^{\prime}, \ldots, x_{n}^{\prime}\right)$ has transcendence degree $n-3$. By Proposition 4.1. the moduli field for $\left(\mathbb{P}_{L_{n}}^{1}, x_{1}, \ldots, x_{n}\right)$ has transcendence degree $n-3$ as well.

\section{Curves With only triple RAmification}

We come to the main result of this paper:

Theorem 5.1. Let $k$ be a field of characteristic $p \neq 3$. For each integer $g \geq 0$, there is finitely generated field extension $k \subset K$ and a smooth stable curve $C$ of genus $g$ over $K$ with the following properties:

(i) The moduli field of $C$ has transcendence degree over $k$ at least $\max (2 g-3, g)$.

(ii) There is a finite generically étale map $C \rightarrow \mathbb{P}_{K}^{1}$ with only triple ramification.

Let me reformulate this over algebraically closed ground fields. First, we extend the result on elliptic curves of Fried, Klassen, and Kopeliovich [2] to positive characteristics:

Corollary 5.2. Suppose $k$ is algebraically closed of characteristic $p \neq 3$. Then for all but finitely many $j$-invariants $j \in k$, the corresponding elliptic curve $E$ admits a finite generically étale map $E \rightarrow \mathbb{P}_{k}^{1}$ with only triple ramification.

The proof is almost the same as for the case of higher genus:

Corollary 5.3. Suppose $k$ is algebraically closed of characteristic $p \neq 3$ and assume $g \geq 2$. Let $S \subset M_{g}$ be closure for the set of all closed points such that the corresponding curve $C$ admits a finite generically étale map $C \rightarrow \mathbb{P}_{k}^{1}$ with only triple ramification. Then we have $\operatorname{dim}(S) \geq 2 g-3$.

Proof. Let $C \rightarrow \mathbb{P}_{K}^{1}$ be as in Theorem 5.1, and choose an integral $k$-scheme $U$ of finite type whose field of rational functions is $K=\kappa(U)$. Shrinking $U$, we may extend $C$ to a smooth relative curve $X \rightarrow U$, and $C \rightarrow \mathbb{P}_{K}^{1}$ to a $U$-morphism $f: X \rightarrow \mathbb{P}_{U}^{1}$. Shrinking further, we may assume that all fibers $f_{u}: X_{u} \rightarrow \mathbb{P}_{u}^{1}$ are generically étale finite maps with only triple ramification.

By Chevalley's Theorem, the image $V=g(U)$ of the classifying map $g: U \rightarrow M_{g}$ is constructible. Shrinking $U$, we may assume that $V \subset M_{g}$ is a subscheme. Since 
the moduli field of $C$ has transcendence degree $\geq 2 g-3$, the dimension of $V$ is at least $2 g-3$. For each rational point $\sigma \in V$, the fiber $g^{-1}(\sigma) \subset U$ contains a rational point because $k$ is algebraically closed, and the result follows.

Proof of Theorem 5.1. First consider the case $g \geq 3$. We shall construct by induction on $n \geq 0$ a pointed smooth stable curve $\left(C_{n}, c_{1}^{n}, \ldots, c_{g-n}^{n}\right)$ of genus $n$ with $g-n$ marked points over some field $K_{n}$, so that the moduli field has transcendence degree $\geq n+g-3$. Moreover, there will be a generically étale finite map $f_{n}$ : $C_{n} \rightarrow \mathbb{P}_{K_{n}}^{1}$ with only triple ramification such that the $c_{i}^{n}$ are ramification points. Induction terminates at $n=g$.

According to Proposition 4.2, the desired curve exists for $n=0$. Suppose we already found by induction $\left(C_{n}, c_{1}^{n}, \ldots, c_{g-n}^{n}\right)$ and $f_{n}: C_{n} \rightarrow \mathbb{P}_{K_{n}}^{1}$ for some $n<g$. The idea now is to trade the last marked point for a genus increase. Conforming with the notation in Section 3 , we set $\tilde{X}_{0}=C_{n}$ and $\tilde{x}=c_{g-n}^{n}$, and let $X_{0}$ be the corresponding cuspidal curve of genus $n+1$ with normalization $\tilde{X}_{0}$. According to Proposition 3.1, there is an effective deformation $X \rightarrow \operatorname{Spec}(A)$ over $A=K_{n}[[t]]$ with closed fiber isomorphic to $X_{0}$. Moreover, the induced map $X_{0} \rightarrow \mathbb{P}_{K_{n}}^{1}$ extends to a family $X \rightarrow \mathbb{P}_{A}^{1}$ whose generic fiber $X_{\eta} \rightarrow \mathbb{P}_{\eta}^{1}$ is a generically étale finite map with only triple ramification. Furthermore, the rational ramification points $c_{1}^{n}, \ldots, c_{g-n-1}^{n} \in X_{0}$ extend to ramification sections over $A$, and define rational ramification points $c_{1}^{n+1}, \ldots, c_{g-(n+1)}^{n+1} \in X_{\eta}$ in the generic fiber.

Let $X^{\prime} \rightarrow \operatorname{Spec}\left(A^{\prime}\right)$ be the semistable reduction for $X \rightarrow \operatorname{Spec}(A)$ constructed in Proposition 1.1. Then we have a morphism $\operatorname{Spec}\left(A^{\prime}\right) \rightarrow \bar{M}_{n+1, g-(n+1)}$. The image of the closed point $0 \in \operatorname{Spec}\left(A^{\prime}\right)$ is a point $\sigma \in \bar{M}_{n+1, g-(n+1)}$ corresponding to the pointed stable curve $\left(C_{n} \cup E, c_{1}^{n}, \ldots, c_{g-(n+1)}^{n}\right)$ of genus $n+1$. Here $E$ is an elliptic curve with $j=0$ and $C_{n} \cap E=\left\{c_{g-n}^{n}\right\}$. It follows that the residue field $\kappa(\sigma)$ has transcendence degree $\geq n+g-3$. As a consequence, the image of the generic point $\eta \in \operatorname{Spec}\left(A^{\prime}\right)$ in $\bar{M}_{n+1, g-(n+1)}$ has residue field of transcendence degree $\geq(n+1)+g-3$.

The residue field of $\eta \in \operatorname{Spec}(A)$ is the field of formal Laurent series $K_{n}((t))$. By [6], Theorem 8.8.2, the curve $X_{\eta}$, the rational points $c_{1}^{n+1}, \ldots, c_{g-(n+1)}^{n+1} \in$ $X_{\eta}$, and the map $X_{\eta} \rightarrow \mathbb{P}_{\eta}^{1}$ are already defined over some finitely generated field extension $K_{n+1} \subset K_{n}((t))$. This gives the desired pointed stable curve $\left(C_{n+1}, c_{1}^{n+1}, \ldots, c_{g-(n+1)}^{n+1}\right)$ of genus $n+1$ with $g-(n+1)$ marked points over $K_{n+1}$, together with the map $f_{n+1}: C_{n+1} \rightarrow \mathbb{P}_{K_{n+1}}^{1}$. We have completed the induction.

It remains to treat the case $g \leq 2$. For $g=0$, we simply take $C=\mathbb{P}_{k}^{1}$ and the identity map $C \rightarrow \mathbb{P}_{k}^{1}$. For $g=1$, we choose $\left(C_{0}, c_{1}^{0}\right)$ without caring for the moduli field, and apply a single deformation argument as above. For $g=2$, we similarly choose $\left(C_{0}, c_{1}^{0}, c_{2}^{0}\right)$ and apply the deformation argument twice.

\section{Connections With Belyi's Theorem}

Belyi's Theorem [1] states that a compact Riemann surface is defined over a number field if and only if it admits a rational function with at most three critical values. Saïdi 11] generalized this to odd characteristics $p \geq 3$. Let me rephrase the part of his result that holds true for all characteristics:

Proposition 6.1. Let $k$ be an algebraically closed field of characteristic $p>0$. 
(i) A smooth proper curve $C$ over $k$ is defined over a finite field if there is a finite map $C \rightarrow \mathbb{P}_{k}^{1}$ with only tame ramification and at most three branch points.

(ii) A smooth proper curve $C$ over $\overline{\mathbb{F}}_{p}$ admits a finite map $f: C \rightarrow \mathbb{P}_{\overline{\mathbb{F}}_{p}}^{1}$ with only tame ramification and at most three branch points if there is at least one finite map $g: C \rightarrow \mathbb{P}_{\mathbb{F}_{p}}^{1}$ with only tame ramification.

Proof. For convenience, I recall Saïdi's argument: The first statement follows from Grothendieck's theory of the tame fundamental group (compare 10, Theorem 6.1). For the second statement, let $h_{n}: \mathbb{P}_{\mathbb{F}_{p}}^{1} \rightarrow \mathbb{P}_{\mathbb{F}_{p}}^{1}$ be the polynomial map $z \mapsto z^{p^{n}-1}$. Then $f=h_{n} \circ g$ is the desired map for some $n$ sufficiently large, as explained in 11, Theorem 5.6.

In characteristic $p \geq 3$, tame functions $g: C \rightarrow \mathbb{P}_{\mathbb{F}_{p}}^{1}$ as in (ii) exist by [3], Proposition 8.1. In characteristic $p=2$, Corollary 5.3 tell us that this holds true for a set of of curves of dimension at least $2 g-3$.

\section{REFERENCES}

[1] G. Belyi: Galois extensions of a maximal cyclotomic field. Izv. Akad. Nauk SSSR Ser. Mat. 43 (1979), 267-276.

[2] M. Fried, E. Klassen, Y. Kopeliovich: Realizing alternating groups as monodromy groups of genus one covers. Proc. Amer. Math. Soc. 129 (2001), 111-119.

[3] W. Fulton: Hurwitz schemes and irreducibility of moduli of algebraic curves. Ann. Math. 90 (1969), 542-575.

[4] A. Grothendieck: Éléments de géométrie algébrique III: Étude cohomologique des faiscaux cohérent. Publ. Math., Inst. Hautes Étud. Sci. 11 (1961).

[5] A. Grothendieck: Éléments de géométrie algébrique IV: Étude locale des schémas et des morphismes de schémas. Publ. Math., Inst. Hautes Étud. Sci. 24 (1965).

[6] A. Grothendieck: Éléments de géométrie algébrique IV: Étude locale des schémas et des morphismes de schémas. Publ. Math., Inst. Hautes Étud. Sci. 28 (1966).

[7] A. Grothendieck: Éléments de géométrie algébrique IV: Étude locale des schémas et des morphismes de schémas. Publ. Math., Inst. Hautes Étud. Sci. 32 (1967).

[8] J. Harris, I. Morrison: Moduli of curves. Grad. Texts Math. 187. Springer, New York, 1998.

[9] L. Illusie: Complexe cotangent et déformations I. Lect. Notes Math. 239, Springer, Berlin, 1971.

[10] F. Orgogozo, I. Vidal: Le théorème de spécialisation du groupe fondamental. In: J.-B. Bost, F. Loeser, M. Raynaud (eds.), Courbes semi-stables et groupe fondamental en géométrie algébrique, pp. 169-184. Progr. Math. 187. Birhäuser, Basel, 2000.

[11] M. Saïdi: Revêtements modérés et groupe fondamental de graphe de groupes. Compositio Math. 107 (1997), 319-338.

[12] S. Schröer: The strong Franchetta Conjecture in arbitrary characteristics. Preprint, math.AG/0203168.

Mathematische Fakultät, Ruhr-Universität, 44780 Bochum, Germany

E-mail address: s.schroeer@ruhr-uni-bochum.de 